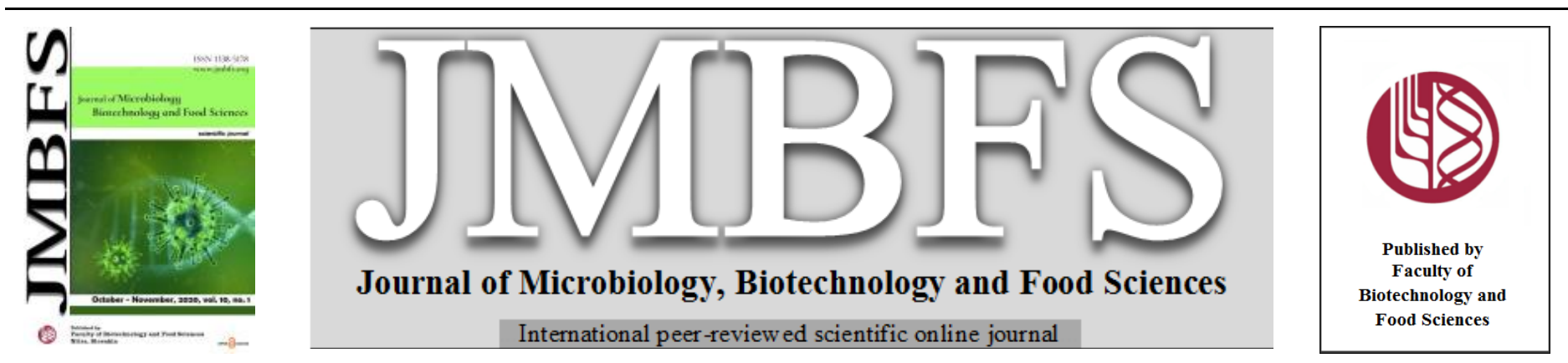

\title{
ANTI-STAPHYLOCOCCAL POTENTIAL OF FUSARIUM SP: FUNGAL ENDOPHYTE ISOLATED FROM RAUWOLFIA SERPENTINA
}

\author{
Lokesh Gambhir ${ }^{1}$, Neha Kapoor ${ }^{2}$ and Sanjai Saxena ${ }^{3 *}$ \\ Address(es): Dr. Sanjai Saxena, \\ ${ }^{1}$ Department of Biotechnology, School of Basic \& Applied Sciences, Shri Guru Ram Rai University, Dehradun, Uttrakhand, 248001. \\ ${ }^{2}$ School of Applied Sciences, Suresh Gyan Vihar University, Jaipur, Rajasthan, 302017. \\ ${ }^{3}$ Department of Biotechnology, Thapar Institute of Engineering \& Technology, Patiala, Punjab, 147004.
}

*Corresponding author: sanjaibiotech@yahoo.com

doi: 10.15414/jmbfs.2020.10.2.235-240

\section{ARTICLE INFO}

Received 30. 3. 2020

Revised 14. 6. 2020

Accepted 18. 6. 2020

Published 1. 10. 2020

$\underline{\text { Regular article }}$

OPEN $\partial_{\text {ACCESS }}$

\begin{abstract}
Fungal endophytes are considered as repository of potent novel bioactive compounds with profuse chemical diversity. These bioactive compounds have been widely utilized in pharmaceutical and agrochemical industries. The present work was undertaken to bio-prospect the fungal endophytes from Rauwolfia serpentina, a medicinal plant used in multiple indigenous preparations, for possible therapeutic interventions. 21 fungal endophytes were isolated from Rauwolfia serpentina with maximum colonization in leaf (42.8\%) followed by bark $(33.3 \%)$ and stem $(23.8 \%)$. In the enzymatic activity screening, \#15RSBNEY was found to be potent amylase producer and \#3RSSTNEY as potential cellulase producer. Further, three isolates viz. \#16RSLBRT, \#10RSLBRT and \#23RSSTNEY exhibited promising antibacterial activity with maximum activity exhibited by \#10RSLBRT against all test strains of Staphylococcus aureus. Interestingly, it was observed that antibacterial activity of \#10RSLBRT increased by two fold after fractionation with acetone and chloroform. Based on above result, the culture filtrate of \#10RSLBRT was extracted with hexane, chloroform and ethyl acetate followed by agar well gel diffusion method for antibacterial activity. The crude ethyl acetate residue of \#10RSLBRT hereby exhibited potent antibacterial activity. The potential isolate i.e. \#10RSLBRT was identified by molecular taxonomic tools using internal transcribed spacer rDNA analysis and was clustered in the Fusarium incarnatum-equiseti complex. Further studies on the characterization of bioactive compounds of ethyl acetate extract of \# 10RSLBRT are warranted for the development of anti-staphylococcal therapy.
\end{abstract}

Keywords: Rauwolfia serpentina, Fusarium sp, Staphylococcus, antimicrobial activity, Molecular taxonomy

\section{INTRODUCTION}

Endophytic fungi are the ingenious collection of microorganisms which resides within the plant tissues, for entire or part of their life cycle with no apparent symptoms of any infection (Saikkonen et al., 1998). Various fungal endophytes have been recognized to encompass potent anticancer, anti diabetic, insecticidal and immunosuppressive agents (Strobel $\boldsymbol{e t}$ al., 1999). The era of discovery of novel therapeutic scaffolds from fungal endophytes was marked with isolation of the anticancer drug, Paclitexel (Taxol) from endophytic fungi Pestalotiopsis microspora, recovered from Himalayan yew tree, Taxus wallichiana, without resulting any visible injury to the host plant (Strobel et al., 2002). Thus, biodiversity of endophytes is an imperative resource of the chemically diversified bioactive compounds based on their relationship with the plants. Endophytic metabolites produced share the same chemistry as their respective host plant and produce the same compound with more bioactive potential (Strobel et al., 2002; Jia et al., 2016). These are recognized as reasonable untapped and prospective resource of novel natural products for utilization in Pharmaceutical and agro industries. The selection of plant species as a source outlines the screening of fungal endophytes and thereby provides huge spectrum of bioactive metabolites having immense potential as pharmacophores. Rationale of plant selection is based upon their pharmaceutical usage and their ecological niche. Tropical and temperate rainforests are the most biologically diverse terrestrial ecosystems on earth and plants growing in these areas are being surveyed for harboring endophytes. Thus, immense potential exists in screening biodiversity of endophytic fungi for exploring novel chemistries possessing enzymatic and antibacterial properties (Uzma et al., 2018).

Natural compounds isolated from medicinally important plants are considered to be conventionally leading agents revealing the unexplored chemical diversity incomparable to the principal combinatorial databases (Rajamanikyam et al., 2017). Rauwolfia serpentina, commonly known as sarpagandha and chandrika, is an ethnomedicinal evergreen shrub of the dogbane or Apocynaceae. It is rich source of indole alkaloids which are available in every part of the plant along with stem and leaves (Yarnell et al., 2004) Reserpine is the most extensively deliberated alkaloid found in $R$. serpentina which is used in traditional indigenous medicinal preparations in India for centuries as a remedy to various problems, including snake bites, hypertension, uterine stimulant, malaria, abdominal pain, and dysentery (Roy, 2010). Extracts of Rauwolfia serpentina has been shown to encompass potent anti-microbial activity against pathogenic Staphylococcus aureus, Salmonella typhi, Salmonella typhimurium, Yersinia enterocolitica, Escherichia coli and Candida albicans (Elizabeth, 2017). However, associated side effects including nausea congestion, lethargy, sedation, hypotension, vomiting, abdominal cramping and gastric ulceration limits its use (Lobay, 2015).

Based on these observations, the present work was undertaken to unravel the diversity of fungal endophytes in Rauwolfia serpentina as a potent source of novel fungal endophytes with bioactive potential for pharmaceutical and therapeutic interventions. Rauwolfia serpentina inhabiting the BRT wildlife sanctuary, Karnataka and Neyyer Wildlife Sanctuary, Kerala was collected for the recovery of endophytic fungi and further prospecting for various enzymatic and antibacterial bioactivities.

\section{MATERIALS AND METHODS}

\section{Sample Collection and isolation of fungal endophytes}

BRT wildlife sanctuary, Karnataka and Neyyer wildlife sanctuary, Kerala were explored for collection of fresh Leaves, stem and bark of Rauwolfia serpentina. The collected plant parts were transported to the laboratory in sealed zip pouches and preserved at $4^{\circ} \mathrm{C}$. The samples were subjected to surface sterilization by treating with $0.1 \%$ sodium hypochlorite for $3 \mathrm{~min}$ followed by $70 \%$ ethanol for $45 \mathrm{sec}$ and $30 \%$ ethanol for $30 \mathrm{sec}$ under aseptic conditions (Mitchell et al., 2001). The pre-sterilized samples were incised into pieces of $1-2 \mathrm{~mm}$ in size under sterilized conditions and were cultured on potato dextrose agar (PDA) plates at $28^{\circ} \mathrm{C}$ for $8-10$ days. The inoculated plates were recurrently examined for 
the emergence of fungal hyphae from inoculated segments. Appearing fungal hyphae were transferred on PDA plates to attain pure culture. The pure active culture was stored on $10 \%$ glycerol-PDA slants (Kapoor and Saxena, 2018)

\section{Screening for extracellular enzyme production}

a) Amylase activity assay- The endophytic isolates were examined for the production of extracellular amylase by culturing them on $2 \%$ soluble starchGlucose Yeast Peptone (GYP) agar medium. The plates were incubated at $28^{\circ} \mathrm{C}$ for $72 \mathrm{~h}$ followed by flooding with staining solution containing $1 \%$ iodine. The zone of clearance appeared around the colony indicated amylase production (Kapoor et al., 2018; Sunitha et al., 2012).

b) Cellulase activity assay- The endophytic fungal isolates were screened for cellulose production on Yeast-Peptone Agar (YPA) medium containing $0.5 \%$ carboxymethyl cellulose (CMC). The plates were incubated at $28^{\circ} \mathrm{C}$ for $72 \mathrm{~h}$. Staining was done using $0.2 \%$ aqueous Congo red dye followed by destaining with $1 \mathrm{M}$ sodium chloride. The zone of clearance appeared around the colony indicated the cellulase activity (Teather and Wood, 2012).

c) Laccase activity assay- The extracellular laccase production by fungal endophytes was screened by inoculating $5 \mathrm{~mm}$ mycelial plug on GYP agar medium containing $0.005 \% \alpha$-naphthol. The plates were incubated at $28^{\circ} \mathrm{C}$ for $24 \mathrm{~h}$. Laccase induced oxidation of $\alpha$-naphthol resulted in the color change of the medium from transparent to blue. Hence formation of blue color around the colony confirms the laccase production (Rao et al., 2019).

\section{Secondary metabolites prodcution}

Broth cultures of isolates were produced by following the method of Rodriguez et al (2000). Briefly, $5 \mathrm{~mm}$ mycelial plug of cultured endophytic fungal isolate was inoculated in Tryptone Soya Broth (TSB), Potato Dextrose broth (PDB), Czpek-Dox broth (CDB), yeast extract-peptone broth (YEPD) medium, PDB:YEPDB (PY) media, PDB:CDB (PC) media, PDB:TSB (PT) media PDB:YEPD:TSB:CDB (PYTC) media followed by incubation at $28^{\circ} \mathrm{C}$ with continuous shaking for 10 days. Post incubation period, the fungal mass was separated from the supernatant to obtained cell free filtrates that were further stored at $-4^{\circ} \mathrm{C}$

\section{Liquid-liquid Extraction}

The culture filtrates of selected fungal endophytes were subjected to extraction with diverse solvent systems viz. Hexane, ethyl acetate, chloroform and acetone The culture filtrate was extracted with each solvent thrice, organic layers were taken out and dehydrated using sodium sulphate. The organic layer so obtained was evaporated to dryness by using Rotatory evaporator. The obtained crude residue was dissolved in Dimethylsulfoxide (DMSO) and preserved at $4^{\circ} \mathrm{C}$ for further use.

\section{Maintenance of Test microorganisms}

The test organisms included bacterial isolates and were broadly grouped into standard and clinical isolates. The clinical isolates included the following organisms: Staphylococcus aureus (G3), Staphylococcus aureus (G26) procured from Government Medical College, Patiala. The standard isolate was Staphylococcus aureus (NCTC 6571) taken from the Microbial type culture collection (MTCC), IMTECH, Chandigarh. The bacterial cultures were maintained on Muller Hinton Broth (MHB) with $2 \%$ glycerol and stored at $4{ }^{\circ} \mathrm{C}$. These cultures were further streaked on Muller Hinton Agar (MHA) plate and incubated at $37^{\circ} \mathrm{C}$ overnight. A single pure colony was transferred to the MHB and incubated $16-18 \mathrm{~h}$ at $37^{\circ} \mathrm{C}$ before being used as test organism. The visual turbidity of the culture was tested against the standard $0.5 \mathrm{McF}$ arland solution.

\section{Kirby-Bauer Antimicrobial Susceptibility Test}

A total of 8 different combinations of crude EA and aqueous extract (PDB, CDB, TSB, YEPDB, PY, PT, PC, PYTC media) were investigated for antibacterial activity by $\mathrm{KB}$ disc diffusion method. A $5 \mathrm{~mm}$ size disc were used having loading capacity of $30 \mu \mathrm{l}$. Fungal extract $(6.4 \mathrm{mg} / \mathrm{ml})$ was loaded in sterile discs under laminar conditions and left for drying. The turbidity of test organism was adjusted with $0.5 \mathrm{McF}$ arland solution followed by swabbing of MHA plates. The loaded discs were placed on MHA plates and incubated at $37^{\circ} \mathrm{C}$ overnight for $18 \mathrm{~h}$ to $24 \mathrm{~h}$. Anti-staphylococcal activity was analyzed by measuring diameter of zone of inhibition in mm (Magaldi et al., 2004). Three independent experiments were performed and the average values of anti-staphylococcal activity were calculated.

\section{Agar well diffusion assay}

The agar well diffusion assay was carried out to assess the anti-staphylococcal activity of the crude solvent extracts. Stock solutions of extracts $(1 \mathrm{mg} / \mathrm{ml})$ were prepared in $10 \%$ DMSO and stored at $-4^{\circ} \mathrm{C}$ till further use. $20 \mu 1$ of test extracts were dispended in $5 \mathrm{~mm}$ wells prepared using a sterile cork borer on $24 \mathrm{~h}$ old $\mathrm{MH}$ agar plate aseptically. Further, the wells were sealed and kept for 20 minutes for solidification. The turbidity of test organism was adjusted with $0.5 \mathrm{McFarland}$ solution followed by swabbing of MHA plates and incubated at $37^{\circ} \mathrm{C}$ overnight for $18 \mathrm{~h}$ to $24 \mathrm{~h}$. The anti-staphylococcal activity was determined by measuring the zone of inhibition around the test and control samples (Magaldi et al., 2004; Valgas et al., 2007). Three independent experiments were performed and the average values of anti-staphylococcal activity were calculated.

\section{Molecular identification of potent endophytic fungus}

\section{PCR Amplification}

The potential fungal endophyte was identified by Molecular tools employing ITS1 and ITS4 primers mediated amplification of ITS1-5.8S-ITS2 region (Felsenstein, 1985; White et al., 1990). Genomic DNA extracted from the endophytic fungal isolates was taken as template along with $0.8 \mu \mathrm{M}$ primer, 0.2 $\mathrm{mM}$ dNTP, $1.5 \mathrm{U}$ Taq polymerase to a final volume of $25 \mu \mathrm{l}$. The reaction mixture was subjected to PCR amplification following 39 cycles and a final extension of 5 mins at $72^{\circ} \mathrm{C}$ (Kapoor and Saxena, 2014). PCR amplicon was purified using Wizard $^{\circledR}$ SV Gel and PCR clean up system kit, Promega, USA following manufacturer's protocol. The Purified PCR product was sent for direct sequencing to Xcelris Labs, Gujarat.

\section{Sequence assembly and Phylogenetic identification:}

Sequencher ver. 5 (www.genecodes.com) was used to edit and verify the attained chromatograms and were submitted in the GenBank under the accession number MH237828. BLAST tool was used to analyze the homology in the consensus sequence. Clustal W in MEGA 5.2 was utilized for obtaining the alignment with the reference taxa (Tamura et al., 2011). The phylogenetic history was determined by the Maximum Parsimony method using the Close-NeighborInterchange algorithm (Arnold $\boldsymbol{e t}$ al., 2005) in which the tree is formed based on random addition of sequences with 20 replicates. The analysis involved 19 nucleotide sequences. Alignment gaps were taken as missing characters Bootstrap analysis (1000 bootstrap) was executed to deduce the consensus tree for the demonstration of evolutionary relationship (Kapoor and Saxena, 2014; González-Menéndez et al., 2018).

\section{RESULTS}

\section{Isolation of endophytic fungi}

Leaves, stem and bark samples of Rauwolfia serpentina were subjected to isolation of fungal endophytes on PDA plates. A total of 21 fungal endophytes were recovered from Rauwolfia serpentina. Each host explant showed disparity in the endophytic fungal colonization (Table 1). The maximum colonization was observed in leaf $(42.8 \%)$ followed by bark $(33.3 \%)$ and stem $(23.8 \%)$. No endophytic fungi were recovered from the stem internal issue of the plant. Isolated endophytes were further sub cultured for preservation of pure colonies.

Table 1 fungal endophytes isolated from Rauwolfia serpentina along with plant part, place of sample collection and bioactivity profiling

\begin{tabular}{cllllll}
\hline S. NO & Culture Code & Plant part & Place of sample collection & \multicolumn{2}{l}{ Bioactivities } \\
& & & & Amylase & Cellulase & Laccase \\
1. & $\# 1$ RSLBRT & Leaves & BRT wildlife sanctuary, Karnataka & - & + & - \\
2. & $\# 3 R S L B R T$ & Leaves & BRT wildlife sanctuary, Karnataka & - & - & - \\
3. & $\# 5$ RSLBRT & Leaves & BRT wildlife sanctuary, Karnataka & - & - & - \\
4. & $\# 7 R S L B R T$ & Leaves & BRT wildlife sanctuary, Karnataka & - & - & - \\
5. & $\# 10 R S L B R T$ & Leaves & BRT wildlife sanctuary, Karnataka & - & - & - \\
\hline
\end{tabular}




\begin{tabular}{|c|c|c|c|c|c|c|}
\hline 6. & \#11RSLBRT & Leaves & BRT wildlife sanctuary, Karnataka & - & - & + \\
\hline 7. & \#15RSLBRT & Leaves & BRT wildlife sanctuary, Karnataka & - & - & - \\
\hline 8. & \#16RSLBRT & Leaves & BRT wildlife sanctuary, Karnataka & - & - & - \\
\hline 9. & \#27RSLBRT & Leaves & BRT wildlife sanctuary, Karnataka & - & - & - \\
\hline 10. & \# 1RSBANEY & Bark & Neyyer wildlife sanctuary Kerala & ++ & + & - \\
\hline 11. & \# 7RSBANEY & Bark & Neyyer wildlife sanctuary Kerala & + & - & ++ \\
\hline 12. & \# 11RSBANEY & Bark & Neyyer wildlife sanctuary Kerala & - & - & \\
\hline 13. & \# 12RSBANEY & Bark & Neyyer wildlife sanctuary Kerala & - & - & + \\
\hline 14. & \# 14RSBANEY & Bark & Neyyer wildlife sanctuary Kerala & - & - & +++ \\
\hline 15. & \# 15RSBANEY & Bark & Neyyer wildlife sanctuary Kerala & +++ & - & - \\
\hline 16. & \# 22RSBANEY & Bark & Neyyer wildlife sanctuary Kerala & - & - & - \\
\hline 17. & \#2RSSTNEY & Stem & Neyyer wildlife sanctuary Kerala & + & - & - \\
\hline 18. & \#3RSSTNEY & Stem & Neyyer wildlife sanctuary Kerala & - & ++ & - \\
\hline 19. & \#4RSSTNEY & Stem & Neyyer wildlife sanctuary Kerala & - & - & - \\
\hline 20. & \#15RSSTNEY & Stem & Neyyer wildlife sanctuary Kerala & - & - & - \\
\hline 21. & \#23RSSTNEY & Stem & Neyyer wildlife sanctuary Kerala & - & - & - \\
\hline
\end{tabular}

Note: poor activity (+);Good activity (++), Excellent activity(+++), No activity (-)

\section{Isolated fungal endophytes exhibited potent enzyme production}

Isolated fungal endophytes were examined for the production of commercially important enzymes including amylase, cellulose and laccase.

In the preliminary screening of fungal endophytes, four isolates were found to be positive for producing amylase. Out of 4 positive isolates, maximum amylase activity was observed in \#15RSBNEY followed by \#7RSBANEY, \#1RSBANEY and \#2RSSTNEY. Similarly, three isolates exhibited promising cellulase activity, of which \#3RSSTNEY was found to be potent cellulase producer. In laccase assay, four isolates showed positive results. \# 11 RSLBRT, \# 12 RSBANEY, \# 14 RSBANEY and \# 7 RSBANEY showed blue color around their colony after 4 days of incubation indicating the production of laccase activity showing the ability of fungal isolates to produce de-lignifying enzyme (Figure 1).
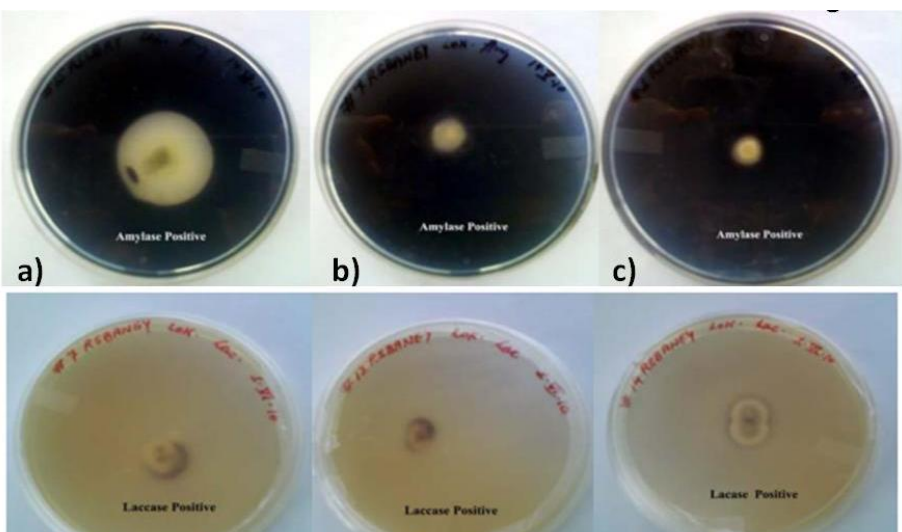

d)

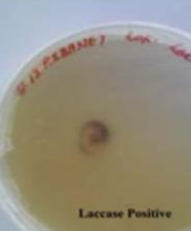

e)
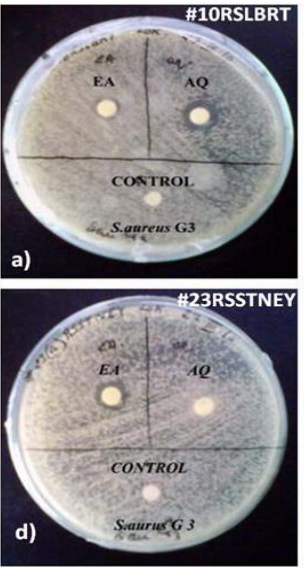

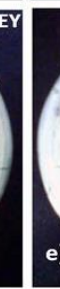

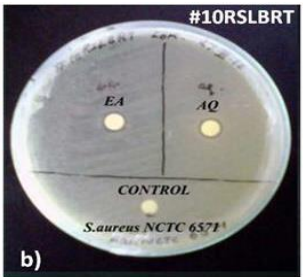

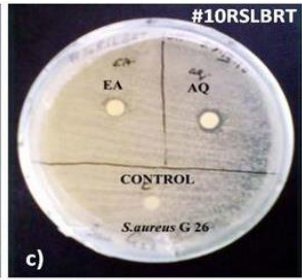

c)

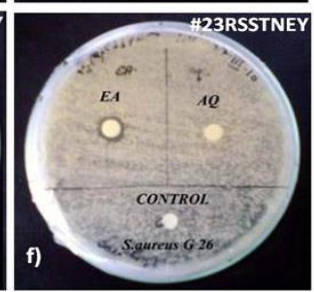

Figure 2 Screening of the antibacterial activity of the isolated endophytes: (A-C) Images represents the $\mathrm{KB}$ disc diffusion assay based anti-bacterial activity of ethyl acetate and aqueous extract of \#10RSLBRT evident from zone of inhibition. Aqueous extract exhibited maximum anti-bacterial activity against pathogenic strains of $S$. aureus (D-F) Images represents the KB disc diffusion assay based anti-bacterial activity of ethyl acetate and aqueous extract of \#23RSSTNEY evident from zone of inhibition. Ethyl activity extract exhibited anti-bacterial activity against pathogenic strains of S. aureus

Aqueous extract of \#10RSLBRT was found to exhibit potent antibacterial action against all test organisms and hence the aqueous spent broth was further analyzed for the optimum $\mathrm{pH}$ needed to exhibit antibacterial activity. A $\mathrm{pH}$ gradient ranging from 4 to 7 was prepared and the extracts were subjected to agar well diffusion method for examining the antimicrobial action. It was observed that maximum activity appeared in range of $\mathrm{pH} 5$ to 6 (Figure 3). Since potent activity was shown by aqueous extract, further fractionation with acetone and chloroform for evaluation of antibacterial activity was carried out. The anti-staphylococcal activity of aqueous layer was observed to get increase by two folds after fractionation with maximum activity against $S$. aureus G3 followed by $S$. aureus G26 and S. aureus NCTC 6571 (Table 3). These results indicated that fractionation with non polar solvents tends to increase the potency of aqueous layer.
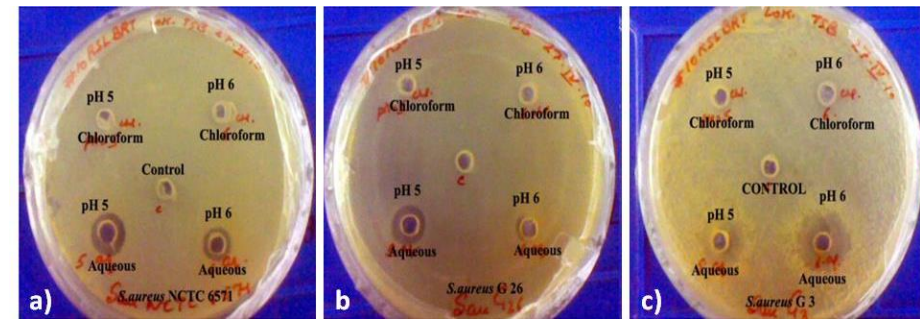

Figure 3 Effect of $\mathrm{pH}$ on anti-staphylococcal activity of \#10RSLBRT: (A-C) Images represent the effect of $\mathrm{pH}$ from 4 to 7 on anti-bacterial potential of the \#10RSLBRT by agar well diffusion assay. Interestingly, $\mathrm{pH}$ range of 5 to 6 showed an increase in the antibacterial activity against $S$. aureus $\mathrm{G} 3$ followed by S. aureus G26 and S. aureus NCTC 6571. 
Table 2 Data represents the zone of clearance in mm obtained from KB disc diffusion assay of selected isolates.

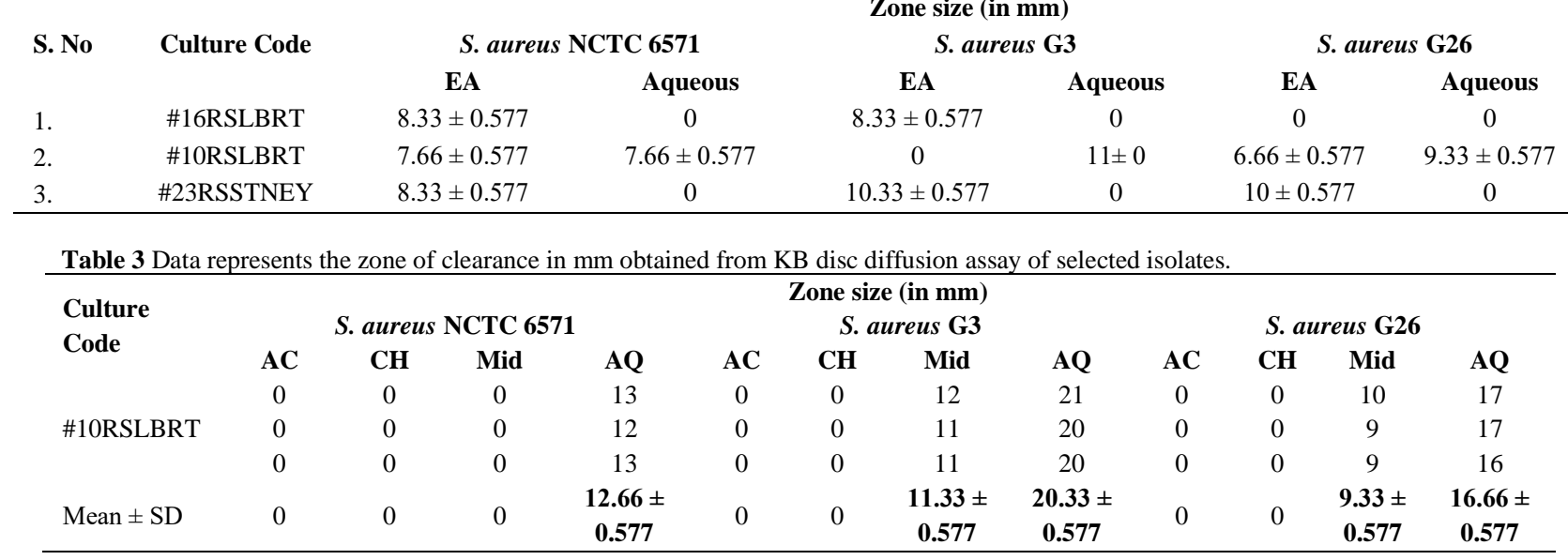

Enhanced anti-staphylococcal activity by solvent fractionation

Based on above observations, it was hypothesized that antibacterial compound resided in aqueous layer may be a strong non polar compound or increasing the non polarity in extraction may channelize the removal of non polar molecules that may hinder in potent antimicrobial activity. To test the proposed hypothesis, hexane was used for fractionation of culture filtrate of \#10RSLBRT followed by extraction with chloroform, Acetone and EA. The agar well gel diffusion assay of obtained extracts revealed that EA and hexane extracts exhibited antibacterial action against all test organisms. Maximum activity was exhibited by EA extract against $S$. aureus NCTC 6571 with zone size of $11.33 \mathrm{~mm}$ (Table 4, Figure 4).

Table 4 Data representing the anti-staphylococcal activity of ethyl acetate and hexane extracts of \#10RSLBRT

\begin{tabular}{|c|c|c|c|c|c|c|}
\hline \multirow{3}{*}{ Culture Code } & \multicolumn{6}{|c|}{ Zone size (in mm) } \\
\hline & \multicolumn{2}{|c|}{ S. aureus NCTC 6571} & \multicolumn{2}{|c|}{ S. aureus $\mathbf{G 3}$} & \multicolumn{2}{|c|}{ S. aureus G26 } \\
\hline & EA & Hexane & EA & Hexane & EA & Hexane \\
\hline \multirow{3}{*}{ \#10RSLBRT } & 12 & 8 & 10 & 7 & 10 & 8 \\
\hline & 11 & 7 & 9 & 7 & 9 & 7 \\
\hline & 11 & 8 & 10 & 7 & 9 & 8 \\
\hline Mean \pm SD & $11.33 \pm 0.57$ & $7.66 \pm 0.57$ & $9.66 \pm 0.57$ & $7 \pm 0$ & $9.66 \pm 0.57$ & $8.66 \pm 0.57$ \\
\hline
\end{tabular}

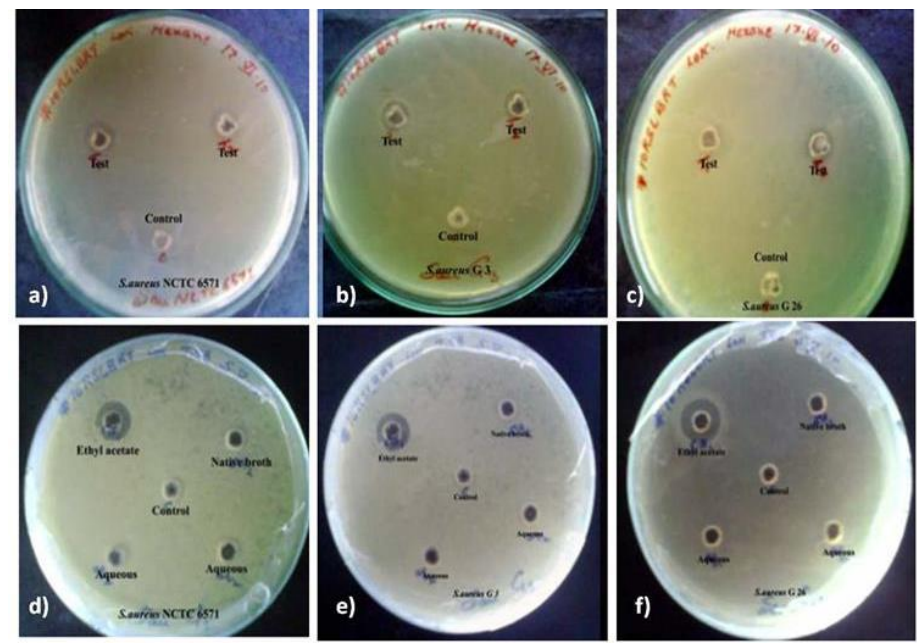

Figure 4 Ethyl acetate extract of \#10RSLBRT exhibited more potent antibacterial activity: To test the possibility of removing the non polar hindering compounds thereby enhancing the antibacterial activity, sequential extraction was done using non polar to polar solvents. (a-c) Images represent the agar well diffusion assay of hexane extracts against $S$. aureus G3 followed by $S$. aureus G26 and S. aureus NCTC 6571. No antibacterial action was professed against the test organisms. (d-f) Images represent the antibacterial activity of ethyl extract post sequential extraction evident from the agar well diffusion assay.

The isolated endophytic fungus was clustered in Fusarium incaranatumequiseti clade by phylogenetic identification.

The BLAST analysis of the sequence data showed close association of the isolate with Fusarium sp. For the speciation of the isolate, Maximum parsimony tree was constructed with consistency index of 0.822917 , retention index of 0.910995 , and the composite index of $0.815983(0.749673)$ for all the sites and parsimonyinformative sites (in parentheses). The tree was divided into 4 different clades of respective Fusarium species viz. Fusarium incarnatum-equiseti complex clade, Fusarium oxysporum clade, Fusarium lateritum clade, Fusarium solani clade. The isolate \#10RSLBRT was clustered in Fusarium incaranatum-equiseti clade thereby confirming its placement in Fusarium incaranatum-equiseti complex (Figure 5). Aspergillus niger was taken as outgroup to root the tree.

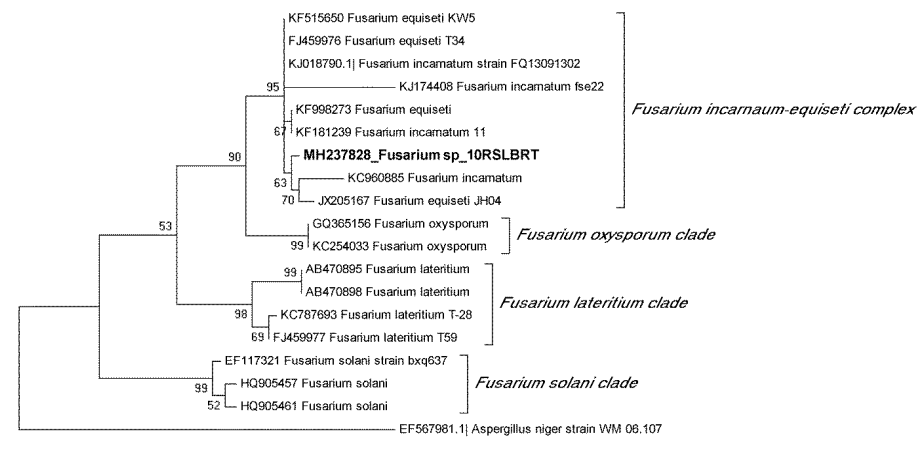

10

Figure 5 The isolated \#10RSLBRT was clustered in Fusarium incaranatumequiseti clade: The Neighbour-joining tree based on the ITS1-5.8S-ITS2 region with the sum of branch length=0.452 is shown. Bootstrap test of 1000 replicates was taken to represent the clustering of associated taxa.

\section{DISCUSSION}

Endophyte inhabiting the host plant acts as defense armor against pathogenic (Strobel and Daisy, 2003). Fungal endophytes have been isolated many plant species including terrestrial plants from different parts like fruits, stems or bark, roots, leaves, tubers, buds, xylem and rachis of grasses, palms, banana, mangroves and halophytes. Fungal endophytes have been the repository of potent bioactive metabolites with diverse action including anti-cancer, antioxidant, antifungal, antibacterial, antiviral, anti-insecticidal and immunosuppressant (Tan and Zou, 2001; Toghueo, 2019). Endophytic fungi are potent source of enzyme producers. Fungal bio-resource is being exploited by researchers in quest of commercially important enzymes like laccase, cellulase, amylase, Lasparaginase etc. For penetrating the host plant and colonizing its tissue, endophytic fungi synthesizes different type of enzymes (Jia et al., 2011) Endophytic fungi are commercially viable as producer of starch digesting enzymes because of efficient conversion of numerous starch sources to sugars without prior gelatinization (Marlida et al., 2015; Toghueo et al., 2017).

Medicinal plants have been the potent repository of novel chemistries destined for pharmaceutical and therapeutic interventions. Recent trends in mycology have focused in isolating novel endophytic fungi from aboriginal medicinal plants for extraction of potent bioactive agents. Rauwolfia serpentina, a medicinal shrub has been studied as a source of endophytic fungal diversity with a colonisation of 18\% (Nath et al., 2017). Also, the isolated endophytes were shown to exhibit 
antibacterial activity. In the present study, Rauwolfia serpentina, a medicinal shrub, was taken as a source of exploring endophytic fungal diversity. Endophytic fungi isolated from the plant were maintained as pure culture and were further assessed for the production of anti-bacterial compound. In all 21 isolates were recovered from different plant parts of Rauwolfia serpentina. Since endophytic fungus have been utilised in production of commercially imperative enzymes, we assessed the isolates for the production of enzymes such as amylase, cellulase and laccase. \# 11 RSLBRT, \# 12 RSBANEY, \# 14 RSBANEY and \# 7 RSBANEY showed potent amylase production. Laccase production was shown by \# 1 RSBANEY, \# 7 RSBANEY, \# 15 RSBANEY and \# 2 RSSTNEY and \# 3 RSSTNEY, \# 1 RSLBRT and \# 1 RSBANEY showed cellulase production. Further, the antibacterial potential of the isolates was evaluated in variety of media composition to decipher the required substrate for potent production of secondary metabolite. Tryptone soya broth as substrate elicited the production of secondary metabolites which was evident with the presence of antibacterial action of the isolates \#16RSLBRT, \#10RSLBRT and \#23RSSTNEY. Since \#10RSLBRT exhibited potent antibacterial action against Staphylococcus aureus viz. NCTC 6571 (control), G3 and G26 (clinical), it was chosen for further evaluation. In sequentially design study the spent broth was fractionated with different solvents and evaluated for antimicrobial action. It was observed that the highest yield and efficacy against $S$. aureus appeared in ethyl acetate extract of \#10RSLBRT endophytic fungal isolate recovered from Rauwolfia serpentina. In agar well diffusion assay it exhibited a zone of inhibition of $11.33 \pm 0.577$ (S.aureus NCTC6571), 9.66 \pm 0.577 and $9.66 \pm 0.577$ against $S$. aureus $\mathrm{G} 3$ and $S$ aureus $\mathrm{G} 26$ respectively. To address the fungal diversity and to determine the identity of the fungus, phylogenetic characterisation of the fungus was performed. The isolated fungus showed close relationship based on sequence similarities of ITS regions with Fusarium sp. Interestingly, the isolate was found to be huddled in Fusarium incaranatum-equiseti clade on building a phylogeneic tree. Fusarium sp. has been documented in the literature to possess potent antimicrobial action (Khan et al., 2018; Zhang et al., 2016).

\section{CONCLUSION}

Staphylococcus aureus is a prime reason of clinical infections in community an healthcare settings. It has a broad spectrum of clinical syndromes from folliculitis to bloodstream infections. It is major causative agent of bacteremia and infective endocarditis. Health associated and surgical site infections are major cause of mortality in clinical settings (. Further the aggravation in the threat of infection is due to the emergence of infections caused by Methicillin Resistan Staphylococcus aureus (MRSA). Thus, discovering novel chemistries as therapeutics against $S$. aureus has kindled the interest of researchers. The present study was aimed at identifying fungal endophytes with anti-staphylococca potential from Rauwolfia serpentina. 21 fungal endophytes were isolated from Rauwolfia serpentina. Three isolates viz. \#16RSLBRT, \#10RSLBRT and \#23RSSTNEY exhibited promising anti-staphylococcal activity with maximum activity exhibited by \#10RSLBRT against all clinical and standard isolates Staphylococcus aureus. Further, \#10RSLBRT was identified and clustered in the Fusarium incarnatum-equiseti complex based on molecular taxonomy. Thus, these results corroborate our hypothesis of isolating potent endophytic fungus from a medicinal shrub Rouwolfia serpentina. The study paves way for bioprospecting fungal endophytes from endangered medicinal plants to exploit the chemical diversity for pharmaceutical interventions. Further studies are required for the purification and characterization of the bioactive compound present in extract of \#10RSLBRT for its potential towards anti-staphylococcal drug development and finding out the mode of action.

Conflict of interest: There is no actual or potential conflicts of interest.

\section{REFERENCES}

Elizabeth (2017). Antimicrobial Activity of Rauwolfa serpentine. Journal of Human Ecology. 12, 375-377. https://doi.org/10.1080/09709274.2001.11907636 Felsenstein, J. (1985). Confidence limits on phylogenies: An approach using the bootstrap. Evolution. 1985; 39:783-791. https://doi.org/10.1111/j.1558 5646.1985.tb00420.x.

González-Menéndez, V., Crespo, G., de Pedro, N. et al. (2018). Funga endophytes from arid areas of Andalusia: high potential sources for antifungal and antitumoral agents. Sci Rep, 8, 9729. https://doi.org/10.1038/s41598-018 $\underline{28192-5}$

Jia, M., Chen, L., Xin, H.L., Zheng, C.J., Rahman, K., Han, T., Qin, L.P. (2016) A Friendly Relationship between Endophytic Fungi and Medicinal Plants: A Systematic Review. Frontiers in Microbiology, 7, 906 https://doi.org/10.3389/fmicb.2016.00906

Kapoor, N., Rajput, P., Mushtaque, M. A., Gambhir, L. (2018). Bio-Prospecting Fungal Endophytes of High Altitude Medicinal Plants for Commercially Imperative Enzymes. Biosc.Biotech.Res.Comm., 11(3), 370-375. http://dx.doi.org/10.21786/bbrc/11.3/4
Kapoor, N., Saxena, S. (2018). Endophytic fungi of Tinospora cordifolia with anti-gout properties. 3 Biotech, 8, 264. https://doi.org/10.1007/s13205-018-1290$\underline{3}$

Kapoor N, Saxena S. (2014). Potential xanthine oxidase inhibitory activity of endophytic Lasiodiplodia pseudotheobromae. Appl Biochem Biotechnol, 173(6), 1360-1374. https://doi.org/10.1007/s12010-014-0927-x

Khan, N., Afroz, F., Begum, M. N., Roy Rony, S., Sharmin, S., Moni, F. Mahmood Hasan, C., Shaha, K., \& Sohrab, M. H. (2018). Endophytic Fusarium solani: A rich source of cytotoxic and antimicrobial napthaquinone and azaanthraquinone derivatives. Toxicology reports, 5, 970-976. https://doi.org/10.1016/j.toxrep.2018.08.016

Kumar, S., Tamura, K., Nei, M. (2004). MEGA3: Integrated software for Molecular Evolutionary Genetics Analysis and sequence alignment. Briefings in Bioinformatics, 5(2), 150-163. https://doi.org/10.1093/bib/5.2.150

Lobay, D. (2015). Rauwolfia in the Treatment of Hypertension. Integrative Medicine: A Clinician's Journal, 14(3), 40-46. PMCID: PMC4566472

Magaldi, S., Essayag, C.M., Capriles, H. et al. (2004). Well diffusion for antifungal susceptibility testing. International Journal of Infectious Diseases, 8, 39-45. DOI: 10.1016/j.ijid.2003.03.002

Mitchell, A., Strobel, G., Moore, E., Robinson, R., Sears, J. (2010). Volatile antimicrobials from Muscodor crispans, a novel endophytic fungus. Microbiology, 156(Pt 1), 270-7. https://doi.org/10.1099/mic.0.032540-0

Nath, A., Chattopadhyay, A., Joshi, S. R. (2015). Biological activity of endophytic fungi of Rauwolfia serpentine Benth: an ethnomedicinal plant used in folk medicines in northeast India. Proceeding of the National Academy of Sciences, 85, 233-240. https://doi.org/10.1007/s40011-013-0184-8

Rajamanikyam, M., Vadlapudi, V., Amanchy, R., Upadhyayula, S. M. (2017) Endophytic Fungi as Novel Resources of natural Therapeutics. Brazilian Archives of Biology and Technology, 60, e17160542. http://dx.doi.org/10.1590/1678-4324-2017160542

Rao, A., Ramakrishna, N., Arunachalam, S. et al. (2019). Isolation, Screening and Optimization of Laccase-Producing Endophytic Fungi from Euphorbia milii. Arabian Journal for Science and Engineering, 44, 51-64. https://doi.org/10.1007/s13369-018-3431-8

Rodrigues, K. F., Manfred, H., Christa, W. (2000). Antimicrobial activity of secondary metabolites produced by endophytic fungi from Spondias mombin. Journal of Basic Microbiology, 40, 261-267. https://doi.org/10.1002/1521 4028(200008)40:4<261::AID-JOBM261>3.0.CO;2-D

Roy, P. (2018). Global Pharma and Local Science: The Untold Tale of Reserpine. Indian journal of psychiatry,60(Suppl 2), S277-S283. https://doi.org/10.4103/psychiatry.IndianJPsychiatry_444_17.

Saikkonen, K., Faeth, S. H., Helander, M., Sullivan, T. J. (1998). Fungal endophytes: a continuum of interactions with host plants. Annual Review of $\begin{array}{llll}\text { Ecology and } & \text { Systematics, } & 29, & 319-343\end{array}$ https://doi.org/10.1146/annurev.ecolsys.29.1.319

Strobel, G., Daisy, B. (2003). Bioprospecting for microbial endophytes and their natural products. Microbiol. Microbiology and Molecular Biology Reviews, 67: 491-502. doi: 10.1128/MMBR.67.4.491-502.2003

Strobel, G. A., Sugawara, F., Harper, J., Hess, W. M. (1999). Oocydin A, a chlorinated macrocyclic lactone with potent anti-oomycete activity from Serratia marcescens. Microbiology, 145, 3557-3564. https://doi.org/10.1099/00221287145-12-3557

Strobel, G. A. (2002). Rainforest endophytes and bioactive products. Critical Reviews in Biotechnology, 22, 3SS15-333. DOI: 10.1080/07388550290789531

Sunitha, V. H., Ramesha, A., Savitha, J., \& Srinivas, C. (2012). Amylase production by endophytic fungi Cylindrocephalumsp. isolated from medicinal plant Alpinia calcarata (Haw.) Roscoe. Brazilian journal of microbiology], 43(3), 1213-1221. https://doi.org/10.1590/S1517-838220120003000049

Tamura, K., Peterson, D., Peterson, N., Stecher, G., Nei, M., Kumar, S. (2011) MEGA5: Molecular Evolutionary Genetics Analysis using Maximum Likelihood, Evolutionary Distance, and Maximum Parsimony Methods. Molecular Biology and Evolution, 28, 2731-9. DOI: 10.1093/molbev/msr121

Tan, R.X., Zou, W.X. (2001). Endophytes: a Rich Source of Functional Metabolites. Natural Product Reports, 18, 448-459. https://doi.org/10.1039/B100918O

Tong, S.Y.C., Davis, J.S., Eichenberger, E., Holland, T.L., Fowler, V.G. Jr. (2015). Staphylococcus aureus infections: epidemiology, pathophysiology, clinical manifestations, and management. Clinical Microbiology Review, 28(3), 603-661. https://doi.org/10.1128/CMR.00134-14

Toghueo, R.M.K. (2019) Anti-leishmanial and Anti-inflammatory Agents from Endophytes: A Review. Natural Products and Bioprospecting, 9(5), 311-328. https://doi.org/10.1080/21501203.2019.1645053

Toghueo, R. M. K., Zabalgogeazcoabc, B. R., Vázquez de Aldanab, Boyoma, F.F. (2017). Enzymatic activity of endophytic fungi from the medicinal plants Terminalia catappa, Terminalia mantaly and Cananga odorata. South African Journal of Botany, 109, 146-153. 10.1016/j.sajb.2016.12.021

Teather, R.M., Wood, P.J. (1982). Use of Congo red-polysaccharide interactions in enumeration and characterization of cellulolytic bacteria from the bovine rumen. Applied and Environmental Microbiology, 43, 777-780. PMCID $\underline{\mathrm{PMC} 241917}$ 
Uzma, F., Mohan, C.D., Hashem, A., Konappa, N.M., Rangappa, S., Kamath, P.V., Singh, B.P., Mudili, V., Gupta, V.K., Siddaiah, C.N., Chowdappa, S., Alqarawi, A.A., Abd Allah, E.F. (2018). Endophytic Fungi-Alternative Sources of Cytotoxic Compounds: A Review. Frontiers in Pharmacology, 9, 309. https://doi.org/10.3389/fphar.2018.00309

Valgas, C., De Souza, S.M., Smânia, E.F.A., Artur, S. (2007). Screening methods to determine antibacterial activity of natural products. Brazilion Journal of Microbiology, 38, 369-380. https://doi.org/10.1590/S1517-83822007000200034 White, T.J., Bruns, T., Lee, S., Taylor, J. (1990). Amplification and direct sequencing of fungal ribosomal RNA genes for phylogenetics. PCR protocols: a guide to methods and applications, New York (NY) Academic Press, 315-22. https://doi.org/10.1016/b978-0-12-372180-8.50042-1

Yarnell, E., Abascal, K. (2004). Treating hypertension botanically. Alternative and Complementary Therapies, 7, 284290. https://doi.org/10.1089/107628001753312121

Marlida, Y., Saari, N., Hassan, Z., Son, R. (2000). Raw starch-degrading enzyme from newly isolated strains of endophytic fungi. World Journal of Microbiology and Biotechnology, 16, 573-578. https://doi.org/10.1023/A:1008935814516

Zhang, H., Sun, X., Xu, C. (2016). Antimicrobial activity of endophytic fungus Fusarium sp. isolated from medicinal honeysuckle plant. Archives of Biological Sciences, 68, 25-30. https://doi.org/10.2298/ABS140401004Z 